\title{
Reactive astrogliosis in epilepsy -passive bystanders no more
}

Volume 5 Issue 4 - 2016

\author{
Keywords: astrogliosis, temporal lobe epilepsy, gfap, $\beta 1$-integrin
}

\section{Introduction}

Temporal lobe epilepsy (TLE) is a neurological disorder that is characterized by spontaneous, recurrent seizures and can be associated with reactive astrogliosis (also known as astrocytosis). This is an adaptive process that consists of alterations in structure and biochemistry of astrocytes and is important for tissue repair and regulation of inflammation. Anti-epileptic drugs (AEDs) used to manage TLE can be associated with refractoriness and substantial side-effects. Most AEDs act through mechanisms that primarily involve neurons; hence, examining the role of reactive astrogliosis in epilepsy could lead to development of novel therapies for epilepsy. The exact role of reactive astrogliosis in epilepsy is not well understood, as experiments suggest both potential pro and antiepileptogenic effects. ${ }^{1,2}$ Given that epilepsy can be associated with neurodegeneration, mossy fiber sprouting and changes in receptor and neurotransmitter function, examining the specific role of reactive astrogliosis in epilepsy has been fraught with difficulties. Previously, a transgenic mouse line was generated ${ }^{3}$ where deletion of $\beta 1$-integrin preferentially in radial glia caused reactive astrogliosis marked by hypertrophy and upregulation of astrocyte-specific markers. Using this transgenic mouse line, in the current Journal of Neuroscience study, ${ }^{4}$ the authors examined whether reactive astrogliosis by itself was sufficient to cause epilepsy, and if so, what are the underlying mechanisms.

In all experiments, control mice were compared to those with reactive astrogliosis caused by deletion of $\beta 1$-integrin in radial glia (' $\beta 1 \%$ mice'). First, reactive astrogliosis was qualitatively and quantitatively confirmed in the cortex of $\beta 1 \%$ mice at various ages. $\beta 1^{-}$ /- mice also had microglial activation; this could be a consequence of reactive astrogliosis as the authors suggest, but could be an independent process as well (http://www.jneurosci.org/content/35/8/3330/ F1.expansion.html). EEG analysis revealed that a subset of $\beta 1 \%$ mice exhibited spontaneous seizures; interestingly, there was a correlation between levels of GFAP and seizure frequency. Interictal spikes (IIS) - the hallmark of an epileptic brain- were also found in $\beta 1 \%$ mice (http://www.jneurosci.org/content/35/8/3330/F2.expansion.html). The authors here examined only convulsive seizures or tonic-clonic seizures. However, analyzing subconvulsive seizures that also occur clinically would have given more complete information about the seizure profile. Since just implanting electrodes can cause seizures due to excitotoxicity, the next question was to examine whether seizures were due to reactive astrogliosis or because of a physical injury to the brain caused by EEG electrodes. Indeed, control mice with implanted electrodes did not show spontaneous seizures, and in $\beta 1^{-}$ I- mice, the incidence of seizures and IIS was unrelated to the extent of damage caused by electrode implantation (http://www.jneurosci.org/ content/35/8/3330/F3. expansion.html). Brain slices from $\beta 1 \%$ mice also exhibited hyperexcitability in pyramidal cells of layer II/III of the cortex in response in two models of in vitro epileptiform activity,

\author{
Sloka S lyengar \\ Department of Neurology, Montefiore Medical Center, USA
}

Correspondence: Sloka S lyengar, Department of Neurology, Montefiore Medical Center, I I I East 2 I th Street, Bronx, NY 1467, 583W, 215th street, Apt A2, New York, USA, Tel (803) 235II 16, Email slokaiyengar2014@gmail.com

Received: October 04, 2016 | Published: December 20, 2016

where a reduced latency to the first ictal and non-ictal event in slices from $\beta 1 \%$ mice was seen. Examination of action potentials in response to current injection and input output $(\mathrm{I} / \mathrm{O})$ curves also showed that Layer II/III pyramidal cells in the cortex of $\beta 1 \%$ mice were more excitable than slices from control mice. But how does one know that these results are specific to astrocytes? To address this, the authors used mice where $\beta 1$-integrin was deleted from neurons instead of radial glia ( $\beta 1 \%$ Nex::Cre mice) and found that these mice did not exhibit hyperexcitability in response to $\mathrm{Mg} 2+$-free aCSF (artificial cerebrospinal fluid: http://www.jneurosci.org/content/35/8/3330/ F4.expansion.html). However, examining whether $\beta 1 \%$ Nex::Cre mice exhibit spontaneous seizures would have been quite informative.

To explore possible reasons underlying epilepsy and in vitro epileptiform activity in $\beta 1 \%$ mice, the authors examined glutamate uptake and homeostasis of $\mathrm{K}+$ and $\mathrm{Cl}$ - ions. Astrocytes play a critical role in tightly regulating $\mathrm{K}+$ homeostasis in the normal brain; indeed increasing $\mathrm{K}+$ concentration is an in vitro model of inducing epileptiform activity. Kir4.1 channels mediate $\mathrm{K}+$ conductance and conditional knockout of Kir4.1 channels in astrocytes is associated with greater propensity to seizures. ${ }^{5}$ In line with this, tissue from people with hippocampal sclerosis was found to have abnormally high level of extracellular $\mathrm{K}+{ }^{6}$ In the current study, $\mathrm{K}+$ homeostasis in $\beta 1 \%$ mice was studied by recording from astrocytes (http://www. jneurosci.org/content/35/8/3330/F5.expansion.html), and parameters of $\mathrm{K}+$ homeostasis showed modest deficiencies in mice with reactive astrogliosis.

Astrocytes are the primary cell type responsible for glutamate uptake ${ }^{7}$ - a single astrocyte can release enough glutamate to depolarize 2-4 adjacent neurons. An increased intracellular glutamate in the hippocampus has been observed in human epileptic tissue. ${ }^{8}$ Here, there were few changes in glutamate transporters in $\beta 1 \%$ mice but glutamate uptake was vastly decreased in $\beta 1 \%$ mice suggesting increased extracellular glutamate as a contributor of hyperexcitability. Glutamine synthetase (GS) - an enzyme necessary for degradation of glutamate into glutamine and for providing precursors for the production of GABA - was reduced in $\beta 1 \%$ mice (http://www.jneurosci. 
org/content/35/8/3330/F6.expansion.html). After exploring excitatory neurotransmission, the authors examined the inhibitory system, as the presence of epileptiform activity could represent an imbalance in GABAergic neurotransmission. GABAergic transmission is regulated by two $\mathrm{Cl}$ - co-transporters $\mathrm{NKCC} 1$ and $\mathrm{KCC} 2$, and alterations in the ratio of these co-transporters so as to confer GABA a depolarizing effect have been shown in neuronal development and epilepsy. ${ }^{9}$ Similarly, in this study, the authors found increased NKCC1 (http:// www.jneurosci.org/content/35/8/3330/F7.expansion.html). Although not examined here, the electrophysiological correlate of this alteration in $\mathrm{Cl}$ - cotransporter ratio would be interesting to see. Administration of bumetanide - a drug that blocks NKCC1 showed a reversal in the seizure phenotype in a subset of $\beta 1 \%$ mice (http://www.jneurosci.org/ content/35/8/3330/F8.expansion.html). In summary, this is the first study to show that global reactive astrogliosis is sufficient to cause seizures, at least partly due to aberrations in glutamate uptake and Clco-transporters. This was found in the absence of blood brain barrier (BBB) compensation, which is important because BBB breach can cause or exacerbate seizures by itself.

Reactive astrogliosis in this study was induced by deleting $\beta 1$ integrin from radial glia at birth. Since radial glia are critical for neuronal migration, examining neuronal migration in $\beta 1 \%$ mice could have helped rule out effects of mismigration on seizures. This is relevant because neuronal mismigration on its own can contribute to seizures e.g. seizures seen in lissencephaly, and due to ectopic granule cells in the hippocampus. Reactive astrogliosis is a hallmark of hippocampal sclerosis - a process typically seen in mesial TLE. In this study, the authors studied the cortex, but investigating the temporal lobe would be quite instructive. This study leads us to ask the question of what would happen if reactive astrogliosis were induced only in a certain area or circuitry of the brain, as opposed to the entire brain as it was done here. Not all mice with reactive astrogliosis showed epilepsy, and only a subset of mice administered bumetanide showed reversal of the seizure phenotype. Why this is the case is not known; however, there is some relevance to the clinical realm, as not everyone with traumatic brain injury develops epilepsy. This study also explains why resecting the sclerotic hippocampus - an area that is invaded by glia - provides seizure reduction. Another question that this study raises is whether stopping reactive astrogliosis would stop epilepsy. Ablation of astrogliosis has been done. ${ }^{10}$ and one could hypothesize that ablation of reactive astrogliosis would be protective against epilepsy. Epilepsy is characterized by not only seizures, but also comorbidities as well. Astrocytes participate in a variety of complex behavior like mood and depression; hence, it could be that reactive astrogliosis plays a role in seizure generation as well as comorbid psychiatric conditions. Astrocytes are coupled by gap junctions and this coupling limits hyperactivity by enabling efficient removal of extracellular glutamate and $\mathrm{K}+$. A breakdown in the gap junction coupling between astrocytes could then cause hyperexcitability and perhaps seizures. A recent study ${ }^{11}$ compared tissue from patients with hippocampal sclerosis (HS) to those without HS, and found that all HS patients showed an uncoupling of astrocytes. In a mouse model of epilepsy, administration of inflammatory mediators was able to cause profound astrocyte uncoupling, which was observed as early as 4 hours post-status epilepticus; hence, early astrocyte uncoupling could possibly predict who with traumatic brain injury develops epilepsy later on. In summary, this Journal of Neuroscience ${ }^{4}$ study provides data suggesting that epilepsy could be viewed as a glial - and not neuronal - dysfunction. Hence, targeting glia could lead to better therapies for epilepsy and associated comorbidites.

\section{Acknowledgments}

None.

\section{Conflicts of interest}

None.

\section{Funding}

None.

\section{References}

1. Faulkner JR, Herrmann JE, Woo MJ, et al. Reactive astrocytes sprotect tissue and preserve function after spinal cord injury. $J$ Neurosci. 2004;24(9):2143-2155.

2. Ortinski PI, Dong J, Mungenast A, et al. Selective induction of astrocytic gliosis generates deficits in neuronal inhibition. Nat Neurosci. 2010;13(5):584-591.

3. Robel S, Mori T, Zoubaa S, et al. Conditional deletion of beta1-integrin in astroglia causes partial reactive gliosis. Glia. 2009;57(15):1630-1647.

4. Robel S, Buckingham SC, Boni JL, et al. Reactive astrogliosis causes the development of spontaneous seizures. $J$ Neurosci. 2015;35(8):3330-3345.

5. Djukic B, Casper KB, Philpot BD, et al. Conditional knock-out of Kir4.1 leads to glial membrane depolarization, inhibition of potassium and glutamate uptake, and enhanced short-term synaptic potentiation. $J$ Neurosci. 2007;27(42):11354-11365.

6. Heinemann U, Gabriel S, Jauch R, et al. Alterations of glial cell function in temporal lobe epilepsy. Epilepsia. 2000;6:S185-S189.

7. Anderson CM, Swanson RA. Astrocyte glutamate transport: review of properties, regulation, and physiological functions. Glia. 2000;32(1):1-14.

8. Petroff O, Errante LD, Rothman DL, et al. Neuronal and glial metabolite content of the epileptogenic human hippocampus. Ann Neurol. 2002;52(5):635-642.

9. Kaila K, Price TJ, Payne JA, et al. Cation-chloride cotransporters in neuronal development, plasticity and disease. Nat Rev Neurosci. 2014;15(10):637-654.

10. Bush TG, Puvanachandra N, Horner CH, et al. Leukocyte infiltration, neuronal degeneration, and neurite outgrowth after ablation of scar-forming, reactive astrocytes in adult transgenic mice. Neuron. 2009;23(2):297-308

11. Bedner P, Dupper A, Hüttmann K, et al. Astrocyte uncoupling as a cause of human temporal lobe epilepsy. Brain. 2015;138:1208-1222. 